\title{
Exotic RG flow of entanglement entropy
}

\author{
Chanyong Park ${ }^{*}$ and Jung Hun Lee๑ ${ }^{\dagger}$ \\ Department of Physics and Photon Science, Gwangju Institute of Science and Technology, \\ Gwangju 61005, Korea
}

(Received 11 January 2020; accepted 13 March 2020; published 6 April 2020)

\begin{abstract}
In this paper, we holographically study the renormalization group (RG) flow in a three-dimensional Einstein-dilaton gravity with a specific scalar potential, which permits several different types of the RG flow with nontrivial beta functions. By using the intrinsic parameter of the potential, we classify the possible holographic RG flows and examine their physical features. Using the Ryu-Takayanagi formulation, furthermore, we investigate how the $c$ function of the entanglement entropy behaves along with the RG flow numerically. We show that the entanglement $c$ function monotonically decreases even in the cases with a nontrivial beta function. For checking the consistency, we also compare the result of the entanglement entropy with the $c$ function derived from the holographic renormalization procedure.
\end{abstract}

DOI: $10.1103 /$ PhysRevD.101.086008

\section{INTRODUCTION}

Among contemporary theoretical physics, holography becomes one of the important research areas. Especially after the AdS/CFT correspondence proposed in [1-4] this conjecture has provided a new tool to understand quantum gravity and nonperturbative quantum features of the quantum field theory. Recently, the entanglement entropy has paid attention to both string theory and condensed matter theory. The entanglement entropy measures the degrees of freedom confined in an arbitrarily chosen spacelike subsystem. In two-dimensional conformal field theories, it has been shown that the coefficient of the universal term in the entanglement entropy is proportional to the central charge representing the degrees of freedom [5-8]. There are many attempts to generalize the two-dimensional result into the higher dimensional cases [9-12]. Despite its salient property, the field-theoretic computation accompanies with a very complicated analysis. In this circumstance, the holographic calculation $[13,14]$ based on AdS/CFT correspondence $[1-4,15,16]$ provides a more tractable tool because it enables us to interpret the entanglement entropy of strongly interacting systems as a geodesic in the dual Einstein gravity. In this work, we investigate the holographic renormalization group ( $R G$ ) flow of the entanglement entropy and the property of the $c$ function along the RG

\footnotetext{
*yong21@gist.ac.kr

junghun.lee@gist.ac.kr
}

Published by the American Physical Society under the terms of the Creative Commons Attribution 4.0 International license. Further distribution of this work must maintain attribution to the author(s) and the published article's title, journal citation, and DOI. Funded by SCOAP ${ }^{3}$. trajectory when the boundary conformal field theory is deformed by a relevant operator [17-35].

The AdS/CFT duality has provided the one-to-one map between nonperturbative conformal field theories (CFT) and gravity/string theories defined in a one-dimensional higher anti-de Sitter (AdS) geometry at least in the large $N$ limit. Surprisingly, it was shown that the holographic calculation of the entanglement entropy can reproduce exactly the same results obtained in a two-dimensional CFT $[13,14]$. However, when we consider a CFT deformed by a relevant operator, the usual CFT description cannot be used anymore at the low energy scale because the relevant deformation spoils the conformal symmetry. In other words, the relevant deformation can dramatically change the UV theory at the IR energy scale. Therefore, a CFT deformed by a relevant scalar operator gives us a chance to find a new CFT at an IR fixed point. To understand such a nontrivial RG flow from the holographic point of view, we consider an Einstein-dilaton gravity with an appropriate dilaton potential. The gravity theory, which admits a smooth interpolation between UV and IR fixed points, has been known not only in gauged supergravity theories in $\mathrm{AdS}_{3}$ but also in other higher dimensional theories [36,37]. Recently, the authors of [38] found new solutions that show exotic behaviors of the RG flow. Related to such exotic RG solutions, in this work we investigate how the dual field theory is modified along with the RG flow by using the holographic renormalization and entanglement entropy techniques.

One of the interesting and important tasks in physics may be counting the number of degrees of freedom of quantum field theories, which decreases monotonically along with the RG flow. This feature called the $c$ theorem is well expressed by a $c$ function depending on the energy scale. 
For a suitably chosen entangling surface, the universal contribution to an entanglement entropy can be matched to the $c$ function $[11,39]$. Such a $c$ function naturally reduces to the central charge at fixed points. Especially, in order to survey the diverse behaviors of $c$ functions for various RG flows, we introduce a specific dilaton potential with one free parameter $a$. Relying on the value of $a$, in this work we show that three different types of the RG flow, for instance, the standard, staircase, and bouncing RG flows [38], are possible. For a positive value of $a$, the standard RG flow naturally appears. For a small negative value of $a$, the staircase RG flow appears and the $\beta$ function described by the dilaton field repeatedly changes its magnitude without changing the sign along the RG trajectory. In a large negative value of $a$, interestingly, we obtain a bouncing RG solution which has a similar feature to the cascading RG flow studied in Ref. [38]. In general, the cascading RG flow violates the Breitenlohner-Freedman (BF) bound at the UV energy scale. However, the bouncing solution we found does not violate the BF bound but changes the sign of the $\beta$-functional iteratively. Another intriguing point we observe is that in the bouncing RG solution, the number of the sign change in the $\beta$ function increases as the absolute value of $a$ becomes large. Despite the nontrivial $\beta$ functions, we find that the $c$ functions of these RG flows always decrease monotonically along with the RG flow.

The rest of this paper is organized as follows: In Sec. II, we discuss the basic setup for computing both the holographic RG flow and the corresponding $c$ function in the Einstein-dilaton gravity. To do so, we introduce the firstorder formalism where the superpotential plays a central role. In Sec. III, we take into account a toy model involving an appropriate potential with one free parameter. In a specific range of the parameter, the theory admits diverse geometric solutions interpolating two AdS spaces that, on the dual field theory side, represent various nontrivial RG flows from a UV to an IR fixed point. We classify the possible RG solutions and investigate their salient features. Applying the RT formulation, in Sec. IV, we study the RG flows of the entanglement entropy with several different parameter values and investigate the corresponding $c$ theorems. Finally, we close this work with some concluding remarks in Sec. V.

\section{HOLOGRAPHIC RG FLOW IN EINSTEIN-DILATON GRAVITY}

In this section, we investigate how an asymptotic AdS geometry deformed by a scalar field is connected to the RG flow and what kind of the RG flow can occur depending on the value of an intrinsic parameter. To do so, let us start by briefly reviewing the holographic RG flow.

Let us consider a Euclidean version of a minimally coupled Einstein-dilaton gravity with an arbitrary scalar potential

$$
\begin{aligned}
S= & -\frac{1}{2 \kappa^{2}} \int d^{d+1} X \sqrt{g}\left(\mathcal{R}-\frac{1}{2} g^{M N} \partial_{M} \phi \partial_{N} \phi-V(\phi)\right) \\
& +\frac{1}{\kappa^{2}} \int_{\partial \mathcal{M}} d^{d} x \sqrt{\gamma} K,
\end{aligned}
$$

where $g_{M N}$ and $\gamma_{\mu \nu}$ indicate a bulk metric and an induced metric on the boundary, respectively. Since the variation of the gravity action usually contains a radial derivative of the metric at the boundary, the Gibbons-Hawking term is usually required to get rid of such a radial derivative term. An extrinsic curvature, $K=g^{M N} K_{M N}$, is given by a covariant derivative of a unit normal vector. Assuming that the dilaton field depends only on the radial coordinate and that the boundary space is flat, the most general metric ansatz preserving the boundary's planar symmetry in the normal coordinate can be represented as

$$
d s^{2}=e^{2 A(y)} \delta_{\mu \nu} d x^{\mu} d x^{\nu}+d y^{2},
$$

where the scale factor $e^{A(y)}$ measures the energy scale of dual field theory and the dilaton field $\phi(y)$ is interpreted as a running coupling of the RG flow. Here, the geometric solution is entirely determined by the scale factor $A(y)$. At a conformal fixed point where the geometry becomes an AdS space, the scale factor is simply $A(y)=-y / R$ where $R$ is the AdS curvature radius. In this description, we implicitly assumed that the asymptotic UV boundary is located at $y=-\infty$, while the IR boundary appears at $y=\infty$. Hence, two fixed points of an RG flow are matched to two boundaries of the above metric ansatz (2.2). The details of the geometry are governed by the equations of motion of $\phi(y)$ and $A(y)$

$$
\begin{aligned}
& 0=2 d(d-1) \dot{A}^{2}-\dot{\phi}^{2}+2 V(\phi), \\
& 0=2(d-1) \ddot{A}+\dot{\phi}^{2}, \\
& 0=\ddot{\phi}+d \dot{A} \dot{\phi}-\frac{\partial V(\phi)}{\partial \phi},
\end{aligned}
$$

where the dot indicates a derivative with respect to $y$. Above the first equation is a constraint, and the second and third are dynamical equations of $A$ and $\phi$. Note that only two of them are independent because combining the first and second equations automatically leads to the third one. As a consequence, Eqs. (2.3) and (2.4) can be regarded as two independent equations determining the geometry up to boundary conditions. It is worth noting that $\dot{A}$ never increases because of $\ddot{A} \leq 0$ in (2.4). From the viewpoint of the holographic RG flow, a holographic $c$ function for $d=2$ is defined as

$$
c=-\frac{3}{2 G \dot{A}},
$$


where the Newton constant is given by $G=8 \pi \kappa^{2}$. Using this relation, the monotonically decreasing behavior of the $c$ function becomes manifest due to (2.4).

In the AdS/CFT, the radial coordinate of the bulk geometry is usually identified with the energy scale of the dual quantum field theory (QFT). This implies that there exists a close relation between the radial motion of the bulk fields and the RG equation of the dual QFT. This holographic relation becomes manifest when we introduce an appropriate superpotential [40-43]. Under the following superpotential:

$$
W(\phi)=-2(d-1) \dot{A},
$$

the previous second order Einstein equations in (2.3) and (2.4) can be decomposed into two first order differential equations,

$$
\begin{aligned}
\dot{\phi}(y) & =W^{\prime}(\phi), \\
V & =\frac{1}{2}\left(\frac{\partial W}{\partial \phi}\right)^{2}-\frac{d}{4(d-1)} W^{2},
\end{aligned}
$$

where the prime indicates a derivative with respect to $\phi$. One can easily check that these two first order equations together with the superpotential in (2.7) are exactly the same as the previous Einstein equations in (2.3) and (2.4). If the boundary position moves from $y=-\infty$ to a finite distance of $y$, this change of the boundary position is associated with the change of the energy scale on the dual field theory side. Since the value of $\phi$ at the boundary is dual to the coupling constant of the dual field theory, Eq. (2.8) represents the energy dependence of the coupling constant on the dual field theory side, which is related to the $\beta$ function representing the RG flow of a coupling constant (see [38,41] for more details),

$$
\beta(\phi) \equiv \frac{d \phi}{d A}=\frac{\dot{\phi}}{\dot{A}}=-2(d-1) \frac{W^{\prime}}{W}
$$

Noting that the superpotential is proportional to the inverse of the $c$ function $(W \sim 1 / c)$, we can easily check that the superpotential does not decrease along the RG flow

$$
\frac{d W}{d y} \geq 0
$$

which is consistent with the $c$ theorem.

\section{VARIOUS RENORMALIZATION GROUP FLOWS}

To study several different kinds of the RG flow, we take into account a toy model having a specific scalar potential

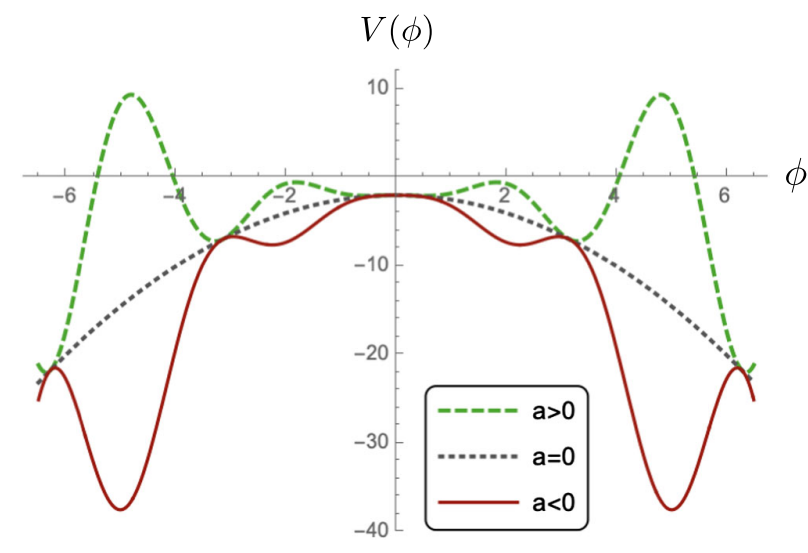

FIG. 1. The potential depending on the parameter $a$.

$$
V(\phi)=-\frac{d(d-1)}{R_{\mathrm{uv}}^{2}}+\frac{m^{2}}{2} \phi^{2}+a \phi^{2} \sin ^{2} \phi,
$$

where $m^{2}=-\Delta(d-\Delta) / R_{\mathrm{uv}}^{2}$ and $a$ is a free parameter. In Fig. 1, we plot the potential with several different parameter values. Above, the first and second terms indicate a negative cosmological constant and a mass of the bulk scalar field, respectively. The last term describes a nontrivial interaction of the bulk scalar field. Notice that, when expanding the potential (3.1) around the UV fixed point $\phi_{\mathrm{uv}_{1}}=0$, the potential gives rise to infinitely many extrema satisfying $V^{\prime}\left(\phi_{*}\right)=0$. Since the potential (3.1) is invariant under $\phi \rightarrow-\phi$, the expansion of the potential is given by

$V^{\prime}(\phi)=\left(\phi-\phi_{\mathrm{uv}_{1}}\right)\left(\phi^{2}-\phi_{\mathrm{ir}_{1}}^{2}\right)\left(\phi^{2}-\phi_{\mathrm{uv}_{2}}^{2}\right)\left(\phi^{2}-\phi_{\mathrm{ir}_{2}}^{2}\right) \cdots$,

where we assume $\phi_{\mathrm{uv}_{1}}<\phi_{\mathrm{ir}_{1}}<\phi_{\mathrm{uv}_{2}}<\phi_{\mathrm{ir}_{2}}<\cdots$. Hereafter, we focus only on the RG flow interpolating the first two fixed points, $\phi_{\mathrm{uv}_{1}}$ and $\phi_{\mathrm{ir}_{1}}$.

Assuming that $\phi$ approaches zero at the boundary, the asymptotic geometry near the boundary is given by a slightly deformed AdS geometry. In this case, $\phi$ and its derivative correspond to a coupling constant (or source) and a vacuum expectation value (VEV) of the dual scalar operator in the dual field theory. Note that the asymptotic AdS geometry can appear only for $-d^{2} / 4<m^{2} R_{\mathrm{uv}}^{2}<0$. In this case, the dual scalar operator becomes a relevant operator. If $m^{2}$ is not in this parameter region, the dual scalar operator is irrelevant. On the gravity side, the corresponding bulk field $\phi$ diverges at the boundary and its gravitational backreaction modifies the asymptotic AdS geometry seriously. For the massless case with $m^{2}=0$, the bulk scalar field is dual to a marginal operator [44-46]. When we focus on the parameter region satisfying $-d^{2} / 4<m^{2} R_{\mathrm{uv}}^{2}<0$, the leading behavior of the asymptotic AdS space is described by 


$$
A(y)=-\frac{y}{R_{\mathrm{uv}}},
$$

where the AdS radius $R_{\mathrm{uv}}$ is associated with the degrees of freedom of the dual UV CFT.

Note that in the asymptotic region, the mass of the bulk scalar field is associated with the conformal dimension, $\Delta$, of the dual scalar operator. The effect of the interaction term of the potential in (3.1) is negligible at least in the asymptotic region because we consider the relevant deformation. Even in this case, in the intermediate and IR regimes the effect of the interaction term becomes important and modifies the AdS geometry seriously into another geometry. In the asymptotic region, anyway, the gravitational backreaction of $\phi$ slightly modifies the asymptotic AdS geometry. In this case, the profile of the scalar field is governed by the following equation of motion:

$$
0=\ddot{\phi}-\frac{d}{R_{\mathrm{uv}}} \dot{\phi}-m^{2} \phi
$$

and its solution is given by

$$
\phi=c_{1} e^{(d-\Delta) y / R_{\mathrm{uv}}}+c_{2} e^{\Delta y / R_{\mathrm{uv}}},
$$

where two integral constants, $c_{1}$ and $c_{2}$, are reinterpreted as a coupling constant (or source) and a VEV of the dual scalar operator, as mentioned before. Substituting the $\phi$ solution (3.5) into Eq. (2.3), the deformed geometry up to higher order corrections reduces to

$A=-\frac{y}{R_{\mathrm{uv}}}-\frac{c_{1}^{2}}{8(d-1)} e^{2(d-\Delta) y / R_{\mathrm{uv}}}-\frac{c_{2}^{2}}{8(d-1)} e^{2 \Delta y / R_{\mathrm{uv}}}$.

Near the UV fixed point with $\phi=0$, we depict the qualitative behavior of the potential relying on $a$ in Fig. 2, which shows that the UV fixed point is unstable regardless of the value of $a$. This means that $\phi$ must roll down to another stable vacuum. Recalling that the value of $\phi$ can be reinterpreted as the coupling constant, the rolling of $\phi$ is

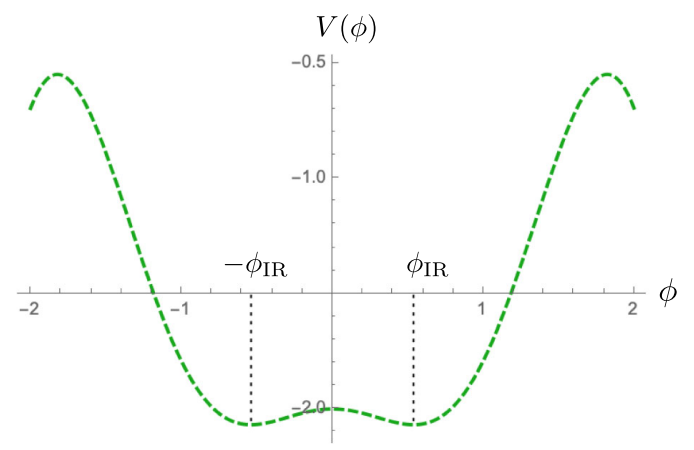

(a) Potential with $a>0$. related to the $\beta$ function discussed before. As shown in Fig. 2, there exist two local minima near $\phi=0$ that correspond to the IR vacuum. The nearest two local minima are always degenerate due to the invariance of the potential under $\phi \rightarrow-\phi$. From now on, we concentrate on the case with $\phi=\phi_{\text {ir }}$ and investigate the RG flow from the UV fixed point with $\phi=0$ to the IR fixed point with $\phi=\phi_{\text {ir }}$. The IR fixed point is stable and has a different vacuum energy $V\left(\phi_{\text {ir }}\right)$ from $V(0)$ at the UV fixed point. On the dual field theory side, the different vacuum energy means that another conformal field theory appears with a different central charge at the IR fixed point. As a result, a geometric solution interpolating two extrema with the rolling $\phi$ describes the RG flow of the dual field theory from the UV to IR fixed points with a nontrivial $\beta$ function. In Fig. 3, we plot numerical solutions satisfying all equations of motion with several different parameter values. Relying on the value of $a$, these numerical solutions can show several different types of RG flow including an exotic RG flow studied in Ref. [38]. Now, we discuss more details about the possible RG flows.

\section{A. Standard and IR incomplete RG flows for $\boldsymbol{a} \geq \mathbf{0}$}

Here, we briefly summarize the standard and IR incomplete RG flows that usually appear in many holographic models.

\section{Standard $R G$ flow}

For $a>0$, the local extremum at $\phi=0$ becomes an unstable local maximum. In this case, a stable local minimum always exists near the local maximum. Therefore, the scalar field $\phi$ naturally rolls down from the local maximum to the other local minimum. On the dual field theory side, this behavior corresponds to the RG flow from the UV to IR fixed points. Moreover, the rolling of $\phi$ is related to the $\beta$ function of the dual field theory. Recalling that the $c$ function monotonically decreases with $c>0$ along the RG flow, we can easily see that $\dot{A}$ always has a negative value, $\dot{A}=-3 /(2 G c)<0$. This feature is also

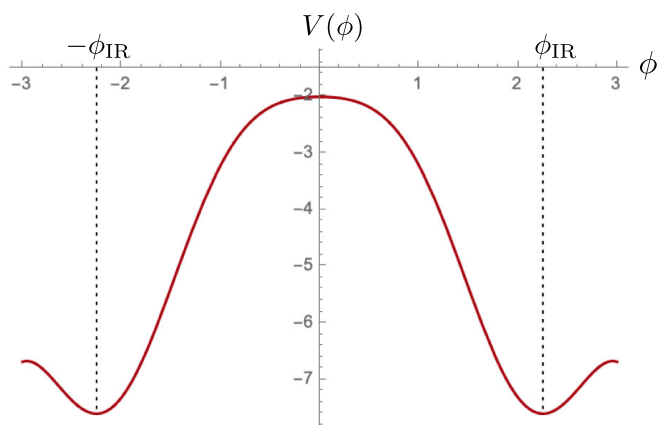

(b) Potential with $a<0$.

FIG. 2. Qualitative behavior of the potential for $d=2$ and $\Delta=1$. 


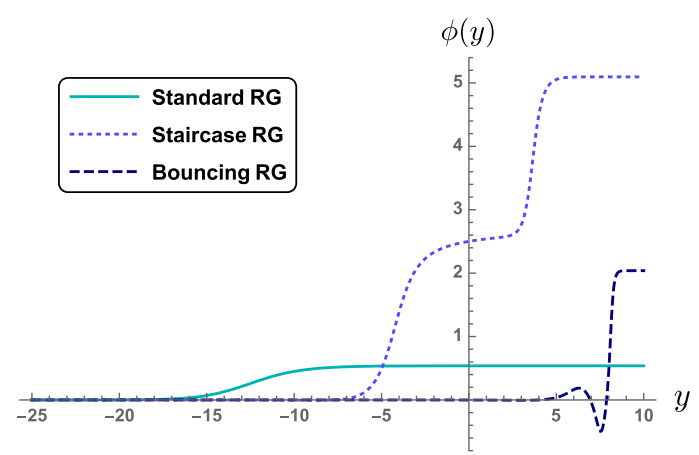

(a)

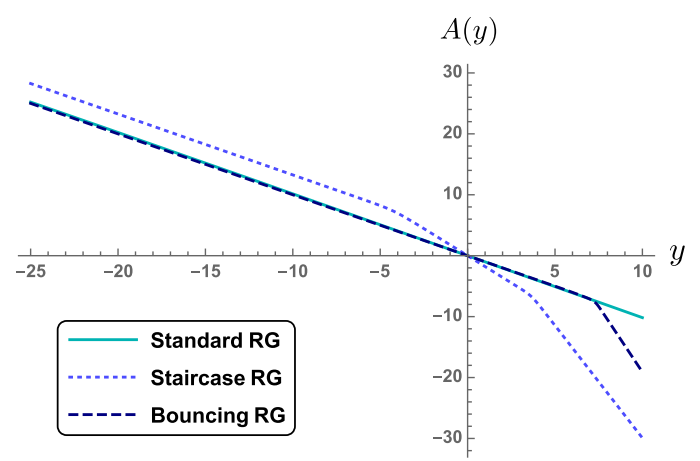

(b)

FIG. 3. The profiles of (a) the scalar field $\phi(y)$ and (b) the metric factor $A$, which represent the standard $(a=1)$, staircase $(a=-0.55)$, and bouncing $(a=-20)$ RG flows, respectively.

manifest in Fig. 3(b). Using this fact, the $\beta$ function in (2.10) can be reexpressed by $\beta \sim-c \dot{\phi}$. If the $\beta$ function is not oscillating during the RG flow, we call it the standard RG flow. The solution for the standard RG flow was constructed by using a variety of potentials (see Ref. [34] and references therein for more details).

\section{Incomplete IR RG flow}

For $a=0$ unlike the case with $a>0$, there is no local minimum near the local maximum at $\phi=0$. In this case, the scalar field rolls down forever and finally diverges in the IR limit. This fact indicates that there is no stable IR fixed point which stops the rolling of the scalar field. From the dual field theory viewpoint, the absence of the local minimum corresponding to the IR fixed point implies the incompleteness of the dual field theory. As a result, the rolling of the scalar field for $a=0$ describes an IR incomplete RG flow of the dual field theory.

\section{B. Exotic (or novel) RG flow for $a<0$}

For $a<0$, a new local minimum near the local maximum again appears. This fact, on the dual field theory side, indicates that there exists a new stable IR fixed point. For $a<0$, however, the RG flow can show a different behavior from the above standard RG flow, which was known as the exotic (or novel) RG flow [38]. Relying on the behavior of the $\beta$ function, the exotic RG flow can be further classified to staircase and bouncing RG flows.

\section{Staircase RG flow}

When $a$ has a small negative value $(a=-0.55)$, the numerical solution in Fig. 3 leads to a staircase RG flow which, at first glance, seems to have several plateaux satisfying $\dot{\phi}=0$. Recalling that $\dot{\phi} \sim W^{\prime}(\phi)$ is related to the $\beta$ function in (2.10), the existence of $\dot{\phi}=0$ implies that additional fixed points except the UV and IR fixed points can exist in the staircase RG flow. In Fig. 4, we depict
$W^{\prime}(\phi)$ and $V^{\prime}(\phi)$ for the standard and staircase RG flows. In the standard RG flow in Fig. 4(a), the UV and IR fixed points corresponding to the ends of the RG flow satisfy $W^{\prime}(\phi)=V^{\prime}(\phi)=0$ simultaneously. In this case, $W^{\prime}(\phi)=0$ means that the $\beta$ function vanishes and $V^{\prime}(\phi)=0$ indicates that the corresponding dual theory is in a stable or unstable equilibrium state. These two conditions are natural requirements to obtain the geometry dual to the ground state of conformal field theories at UV and IR fixed points. In Fig. 4(b), however, the staircase RG flow, as mentioned before, looks to allow an additional fixed point satisfying $W^{\prime}(\phi)=V^{\prime}(\phi)=0$ in the intermediate region of the RG flow. To check whether such an additional fixed point really exists, we zoom in the region around the origin of Fig. 4(b) and plot the result in Fig. 4(c). Figure 4(c) shows that there is no point satisfying $W^{\prime}(\phi)=V^{\prime}(\phi)=0$ simultaneously except for the UV and IR fixed points.

The absence of an additional fixed point discussed above becomes more manifest when we consider the $\beta$ function proportional to $W^{\prime}$. We depict the $\beta$ function as a function of $\phi$ in Fig. 5, where there is no fixed point with a vanishing $\beta$ function except two UV and IR fixed points. In other words, the $\beta$ function does not change its sign during the $\mathrm{RG}$ flow. It is worth noting that the $\beta$ function appearing in Fig. 5 always has a negative value except for the two fixed points. This fact means that the coupling constant of the dual field theory monotonically increases along with the RG flow. Even in this case, since the magnitude of the $\beta$ function oscillates, the coupling constant repeats the fast and slow increasing during the RG flow. We called this type of the RG flow the staircase RG flow.

\section{Bouncing RG flow}

For a negatively large value of $a(a=-20$ in Fig. 3), the profile of $\phi$ in Fig. 3(a) shows totally different behavior from the above staircase RG flow. $\phi$ is monotonically increasing in the staircase RG flow, whereas it oscillates in the case with a negatively large $a$. Since $\dot{\phi}$ is proportional to 


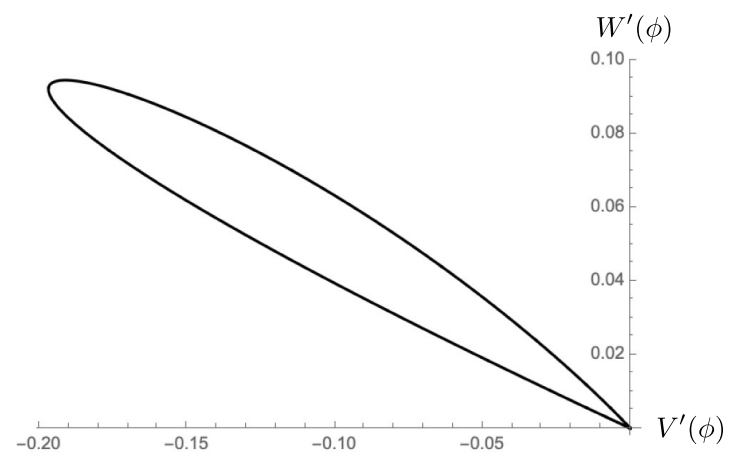

(a) Phase curve in the standard RG flows

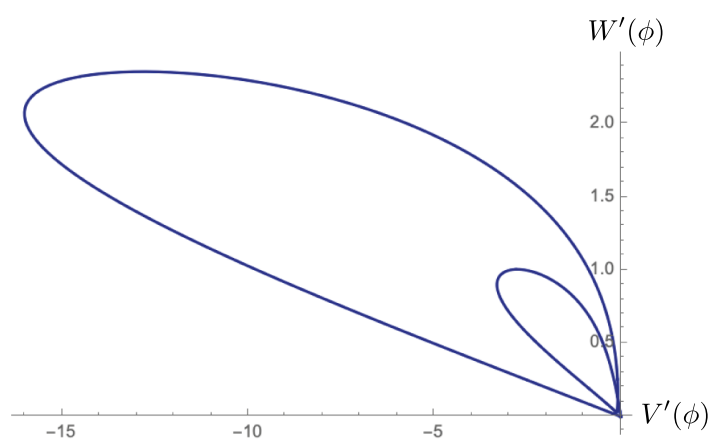

(b) Phase curve in the staircase RG flows

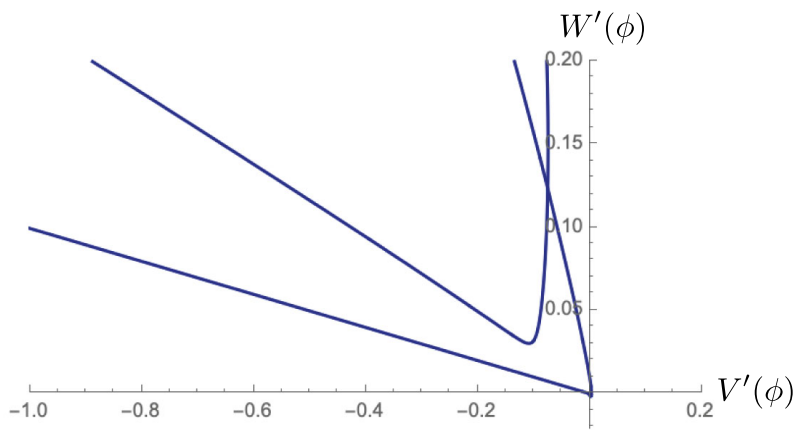

(c) Phase curve near the origin of (b)

FIG. 4. Phase diagram with (a) $a=1$ and (b) $a=-0.55$, respectively. The two curves (a) and (b) flow counterclockwise starting from the origin and terminate their flowing at the same origin. Notice that we distinguish the original point as the UV and the IR fixed points by mapping it into the field or holographic coordinate space. One can easily find that except for the origin there are no intersection points $\left(W^{\prime}=0, V^{\prime} \neq 0\right)$ in the phase space.

the $\beta$ function, the oscillation of $\phi$ indicates that there exists a point with a vanishing $\beta$ function at which the $\beta$ function changes the sign unlike the staircase RG flow. This type of RG flow was known as the bouncing RG flow [38].

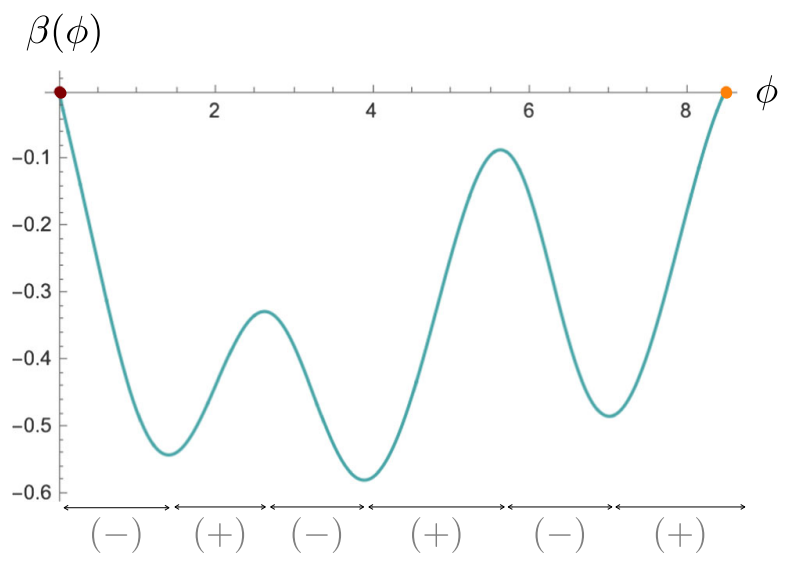

FIG. 5. The $\beta$ function of the staircase RG flow where two ends of the RG flow denote the UV and IR fixed points.
To understand more details of the bouncing RG flow, we introduce a new function by

$$
B(\phi)=\sqrt{-\frac{4(d-1)}{d} V(\phi)} .
$$

This new function is well defined only for $V \leq 0$. In the present work, we consider the RG flows represented by $\phi$ in the range of $\phi_{u v} \leq \phi \leq \phi_{i r}$, so that the value of $V$ during the RG flow always has a negative value satisfying $V\left(\phi_{i r}\right)<V\left(\phi_{u v}\right)<0$. Therefore, the $B(\phi)$ we introduced is a well-defined positive function in the entire range of the RG flow. Rewriting $W^{\prime}(\phi)$ in terms of the new positive function $B(\phi)$ allows two possible branches

$$
W^{\prime}(\phi) \equiv \frac{d W}{d \phi}= \pm \sqrt{\frac{2 d}{d-1}\left(W^{2}-B^{2}\right)} .
$$

At this stage, there are several remarkable points we should note. First, the relation we obtained restricts the range of $W$ to the case of $W \geq B$ because the inside of the 
square root must be non-negative. Second, $W^{\prime}$ vanishes at $W=B$ and two possible branches are smoothly connected at least up to the first order derivative. Suppose that the gravity solution has a point satisfying $W=B$ in the intermediate range of $y$. Then, one branch's solution must smoothly change into the other branch's solution because the region satisfying $W^{2}<B^{2}$ is forbidden. This feature makes the RG flow bounce back at $W=B$ and leads to a vanishing $\beta$ function. Because of these reasons, the RG flow showing this feature is called the bouncing RG flow. In Refs. [32, 38, 47], the authors explored the exotic RG flows in different models and showed that bouncing solutions can have vanishing $\beta$ functions unlike the standard and staircase RG flows, as explained before. Last, although $W^{\prime}=0$ at the bouncing point leads to a vanishing $\beta$ function, it does not guarantee $V^{\prime}=0$. In general, the point with $W^{\prime}=0$ is not coincident with the point satisfying $V^{\prime}=0$ except for the UV and IR fixed points. In Fig. 6, we depict the curves of $W^{\prime}$ and $V^{\prime}$ for $a=-10$ where two bouncing points with $W^{\prime}=0$ exist. However, these bouncing points do not satisfy $V^{\prime}=0$ in the intermediate region, $\phi_{u v}<\phi<\phi_{i r}$. In summary, the bouncing RG flow is the RG flow having the bouncing points, where the $\beta$ function vanishes, but it generally does not lead to additional conformal fixed points due to $V^{\prime} \neq 0$. Then, what is the meaning of $\beta=0$ in the bouncing RG flow? A vanishing $\beta$ function in the bouncing RG flow is directly related to the change of the $\beta$ function's sign. This fact implies that the energy dependence of the coupling constant dramatically changes at the bouncing points. To understand this point more precisely, we need to remember that the positive or negative $\beta$ function usually means that the coupling constant decreases or increases along with the RG flow, respectively. Therefore, the existence of a bouncing point at an intermediate energy scale indicates that a coupling constant increasing along the RG flow starts to decrease after passing through the bouncing point, or vice versa. In other words, the interaction strength of the bouncing RG flow does not monotonically increase along with the RG flow.

So far, we discussed several different types of the RG flow relying on the value of $a$. Especially, the staircase RG flow appears as shown in Fig. 3 for $a=-0.55$. On the other hand, we showed that the bouncing RG flows occur for $a=-20$ in Fig. 3 and for $a=-10$ in Fig. 6. Now, we ask how many bouncing points exist in the bouncing RG flow. Although this question is very interesting, unfortunately answering this question looks very difficult because finding the number of bouncing points requires highly nontrivial nonperturbative analysis. We leave this issue to future work. In this work, instead, we discuss the qualitative relation between the bouncing number and the intrinsic parameter $a$ by using the numerical analysis. In Fig. 7, we depict the numerical behaviors of $\phi$ and $\phi^{\prime}$ in the parameter region of the bouncing flow. For the bouncing RG flow in Fig. 7, a starting point $\left(\phi \neq 0\right.$ and $\left.\phi^{\prime}=0\right)$ and an ending point $\left(\phi=0\right.$ and $\left.\phi^{\prime}=0\right)$ correspond to the UV and IR fixed points, respectively. Except for these two conformal fixed points, Fig. 7 shows that there exist several points satisfying $\phi^{\prime}=0$ in the course of the RG flow. Those additional points exactly correspond to the bouncing points of the bouncing RG flow. Intriguingly, the numerical result in Fig. 7 indicates that the number of the bouncing points increases when the absolute value of the parameter $a$ increases in the bouncing RG flow region.

Before closing this section, there are some remarks on the relation between the bouncing RG flow and the cascading RG flow, which is another possible RG flow solution [38]. In general, the cascading RG flow shows a very similar flowing behavior to the bouncing RG flow,

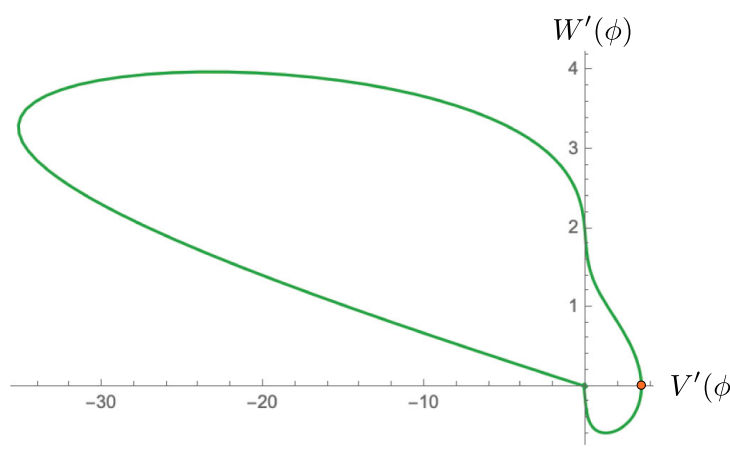

(a)

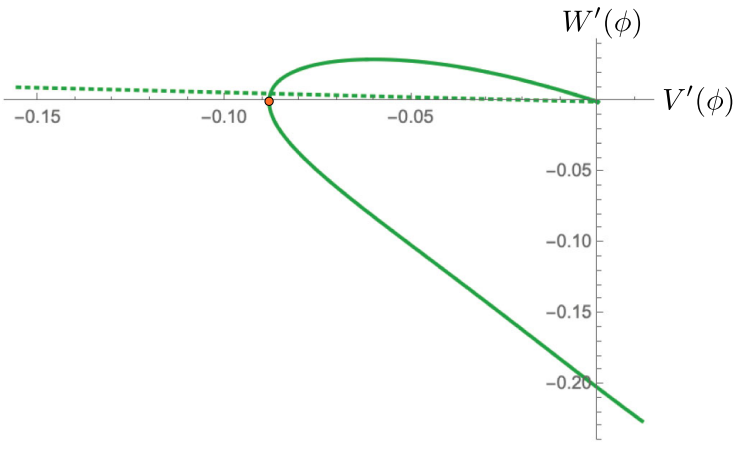

(b)

FIG. 6. Phase diagram with $a=-10$. The two curves (a) and (b) represent two bouncing points. The phase curve starts at the UV fixed point (the origin $W^{\prime}=V^{\prime}=0$ ) and flows counterclockwise and regresses into the origin (the IR fixed point). In the course of a flowing, the phase curve intersects the horizontal line $W^{\prime}=0$ twice (the orange circles). The function $W^{\prime}$ at the intersection point changes its sign: $+\Rightarrow-$ in (b), $-\Rightarrow+$ in (a), which implies that at the critical $\phi_{B}\left[W^{\prime}\left(\phi_{B}\right)=0, V^{\prime}\left(\phi_{B}\right) \neq 0\right]$ the RG flow is bounced and inverts its direction. The dotted curve in (b) represents an ingoing flow to the origin. 


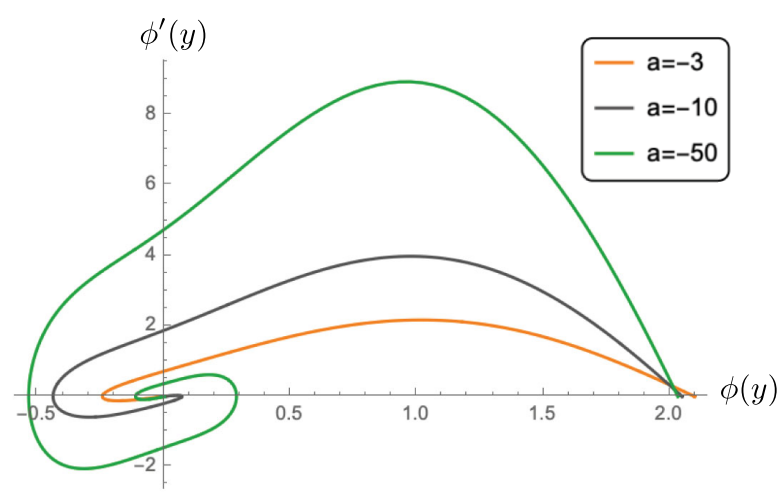

(a)

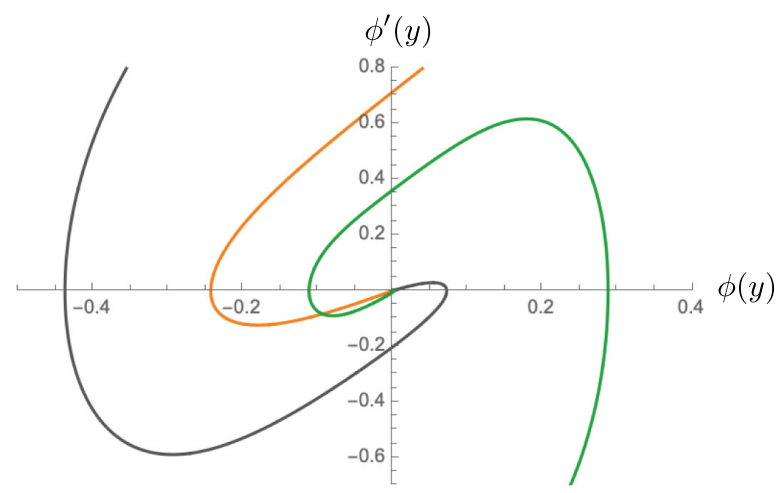

(b)

FIG. 7. Phase diagrams with respect to various negative values of $a$. Each curve has a different number of critical field points $\phi_{B}$ (intersection point, $\phi \neq 0$ and $\left.\phi^{\prime}=0\right)$ : one $(a=-3)$, two $(a=-10)$, and three $(a=-50)$. From a numerical analysis, we find some regularity in which the number of the critical point increases when growing the negative value of $a$. The phase curve starts at the UV fixed point (the origin $\phi=0$ ) and, after clockwise flowing, it stops at the IR fixed value of $\phi$.

though the cascading RG flow usually has an infinite number of the bouncing points. Despite the similar flowing behavior, the cascading and bouncing RG flows have a big difference, which is the reason why a cascading RG flow does not appear in the present model. To understand this important difference between the two RG flows, let us discuss the cascading RG flow more. For the cascading RG flow, it has been well known that there is no well-defined AdS space at a UV energy scale because the cascading RG flow does not allow a unitary CFT at a UV fixed point. On the dual gravity side, the scalar field representing the cascading RG flow usually violates the BF bound and then exhibits an oscillating behavior (tachyonic instabilities $[38,48])$ in the asymptotic region

$$
\Phi(y) \simeq \alpha e^{d y / 2} \cos \left(\frac{|\nu|}{2} y+\gamma\right), \quad y \rightarrow-\infty,
$$

where $\nu=\sqrt{4 m^{2} R_{\mathrm{uv}}^{2}+d^{2}}$ and $\alpha$ and $\gamma$ are integration constants. This is the typical feature usually appearing in an irrelevant deformation. The similar phenomenon without a bouncing behavior was studied in the $\mathcal{N}=1$ supergravity in type IIB string theory [49]. For the bouncing RG flow described by a relevant deformation, the corresponding scalar field is rapidly suppressed in the asymptotic region, so that its gravitational backreaction is usually negligible. However, the scalar field corresponding to the cascading RG flow is not suppressed in the asymptotic region, so that its gravitational backreaction inevitably modifies the asymptotic geometry. Therefore, the asymptotic geometry appearing in the cascading RG flow is not an AdS space. In other words, the cascading RG flow is UV incomplete similar to the $T \bar{T}$ deformation of a two-dimensional IR CFT [50-53]. On the other hand, since the bouncing RG flow is described by a relevant deformation, the dual field theory usually has a well-defined CFT at a UV fixed point.

\section{RG FLOW OF THE ENTANGLEMENT ENTROPY}

The $c$ theorem, as mentioned before, claims that the $c$ function representing the degrees of freedom of a system monotonically decreases along with the RG flow. More accurately, there are three distinguished versions of the $c$-theorem conjecture [54]. (1) The weakest version concerns the degrees of freedom only at the two end points of the RG flow such that $c_{\mathrm{uv}}>c_{\mathrm{ir}}$. (2) A stronger version asserts that $c$ is a monotonically decreasing function along with the entire RG flow. (3) The strongest one claims that the RG flow is a gradient flow of the $c$ function. The last one remains to be proven. Now, we focus on the first and second versions.

In the previous sections, we discussed several different types of the RG flow that allows a nontrivial $\beta$ function. For the bouncing RG flow, in particular, the sign of the $\beta$ function can have both positive and negative values. This fact implies that the interaction strength of the dual field theory repeats increasing and decreasing successively. In this case, it would be interesting to ask how the $c$ function is affected by the change of the interaction strength and how the $c$ function evolves along with the RG flow. In this section, we investigate the change of the $c$ function by using the holographic entanglement entropy.

Except for the free theories with a small perturbation, in general, it is a very difficult task to calculate the entanglement entropy of an interacting field theory analytically. Even in this case, the holographic technique based on the AdS/CFT correspondence provides a very prominent tool that is useful to understand nonperturbative features of strongly interacting systems. According to the Ryu-Takayanagi (RT) 
proposition [13], the entanglement entropy of the dual field theory has a one-to-one map to the area of the minimal surface extended to the bulk geometry. Now, we investigate the evolution of the entanglement entropy along with the RG flow by using the RT formula. To do so, we assume that the entangling points are located at $x= \pm l / 2$. Then, a system is divided into a subsystem with $-l / 2 \leq x \leq l / 2$ and its complement. In this case, if the dual geometry is described by (2.2), the area of the minimal surface is determined by

$$
S_{E}=\frac{1}{4 G} \int_{-l / 2}^{l / 2} d x \sqrt{e^{2 A(y)}+y^{\prime 2}},
$$

where $y$ is given by a function of $x$. Because of the invariance of the action under $x \rightarrow-x$, the minimal surface must have a turning point denoted by $y_{*}$ where $y^{\prime}$ vanishes. After solving the equation of motion, the size of the subsystem can be reexpressed in terms of the turning point

$$
l=2 \int_{y_{*}}^{\infty} d y \frac{e^{A_{*}}}{e^{A} \sqrt{e^{2 A}-e^{2 A_{*}}}},
$$

where $A_{*}$ is the value of $\mathrm{A}$ at $y=y_{*}$. In addition, the entanglement entropy can also be rewritten as an integral form with the turning point

$$
S_{E}=\frac{c}{3 R} \int_{y_{*}}^{\epsilon_{\mathrm{uv}}} d y \frac{e^{A}}{\sqrt{e^{2 A}-e^{2 A_{*}}}},
$$

where we introduce an appropriate UV cutoff $\epsilon_{\mathrm{uv}}$ to regulate a UV divergence and $c=3 R / 2 G$ means the central charge of a UV CFT. The UV central charge, as shown in the above formula, appears as an overall multiplication factor, so that the exact value of $c$ has nothing to do with the qualitative behavior of the RG flow. Because of this reason, from now on, we take $c=1$ and focus on the qualitative feature of the entanglement entropy RG flow.

After performing the above two integrals and rewriting the entanglement entropy in terms of the subsystem size $l$, we finally obtain the entanglement entropy in the UV region

$$
S_{A}=\frac{c}{3} \log \frac{l}{\epsilon_{\mathrm{uv}}}+\delta(l)
$$

where $\delta(l)$ is a function depending on the subsystem size. This is exactly the form expected from a two-dimensional CFT [6]. Here, the first term is crucially relying on the UV theory from the RG flow viewpoint. If we consider a relevant deformation of a UV CFT such as the various RG flows studied in this work, the first term always appears because the relevant deformation does not affect the UV theory. However, the second term above is not universal but crucially depends on the deformation.
To understand the IR physics beyond the UV regime, a nonperturbative method is required. If we are interested in the degrees of freedom of the IR physics, it can be represented as a holographic $c$ function based on the gauge/gravity duality. Using the holographic entanglement entropy, the $c$ function of a two-dimensional QFT is defined by $[17,18]$

$$
c=3 \frac{d S_{E}(l)}{d \log l}=3 l \frac{d S_{E}(l)}{d l},
$$

where $S_{E}(l)$ denotes the entanglement entropy evaluated with the subsystem size $l$. In this case, the subsystem size is reinterpreted as the inverse of the energy scale observing the system so that the system's energy scale moves from the UV to the IR region when the subsystem size increases. This is exactly the same feature of the RG flow not in the momentum space but in real configuration space. One important thing we need to note is that the $c$ function must reduce to the central charge of a CFT at a conformal fixed point.

In a deformed $\mathrm{AdS}_{3}$ space, in general, the $c$ function can be formally rewritten as [55]

$$
c=\frac{3 d S_{E}}{d \log l}=\frac{3}{4 G} \frac{l}{\gamma(l)},
$$

where $\gamma(l)$ is related to a conserved quantity appearing in (4.1). Since the holographic entanglement entropy in (4.1) does not explicitly depend on $x$, there is one conserved quantity which at the turning point is given by

$$
\gamma\left(y_{*}\right)=e^{-A_{*}} .
$$

In this case, since the position of the turning point crucially relies on the subsystem size, $\gamma\left(y_{*}\right)$ can be represented as a function of $l$ instead of $y_{*}$ by using (4.2). In Fig. 8, we plot several exact $c$ functions appearing in the standard, staircase, and bouncing RG flows with different values of $a$. The result shows that the $c$ function always monotonically decreases independent of the type of the RG flow. For the bouncing RG flow with both positive and negative $\beta$ functions, the numerical result shows that the $c$ function always decreases monotonically along with the RG flow regardless of the strength of the interaction.

For a consistency check, in Fig. 9 we also compare the $c$ function in (4.6), which was derived from the holographic entanglement entropy, with (2.6) obtained in the holographic renormalization procedure. The result in Fig. 9 shows that two $c$ functions determined by two different ways have a small discrepancy in the intermediate energy scale. Nevertheless, these two $c$ functions shows the qualitatively same flowing behavior in the entire energy scale and exactly reduces to the central charges of the UV 


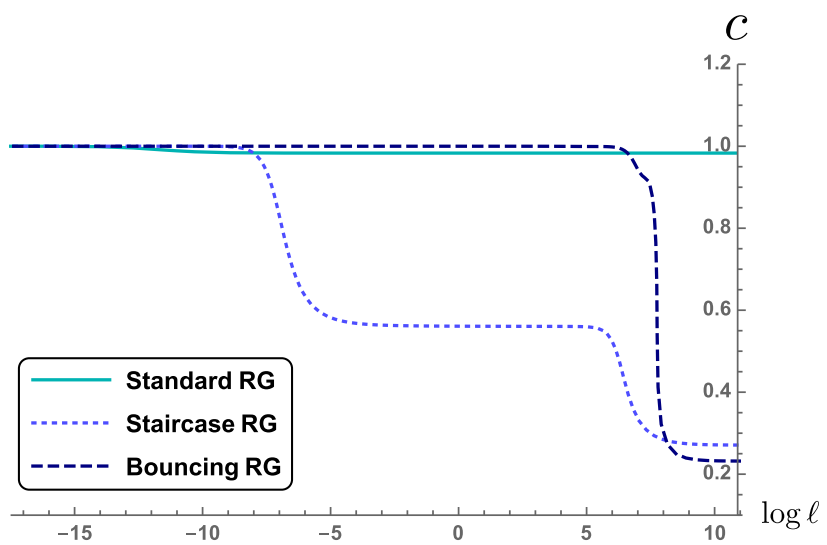

FIG. 8. The entropic $c$ theorems (4.6) evaluated in the $\mathrm{AdS}_{3}^{\mathrm{uv}} \Rightarrow$ $\mathrm{AdS}_{3}^{\text {ir }} \mathrm{RG}$ flows with different values of $a$. The reference central charge at the UV fixed point, $c_{\mathrm{uv}}=1$. The values of $c_{\mathrm{ir}}$ are 0.9833 (standard RG), 0.2708 (staircase RG), and 0.2319 (bouncing RG), respectively.

and IR CFT at the fixed points. As a consequence, although two $c$ functions calculated here have a small quantitative difference, they are qualitatively almost equivalent and represent the RG flow expected by the $c$ theorem.

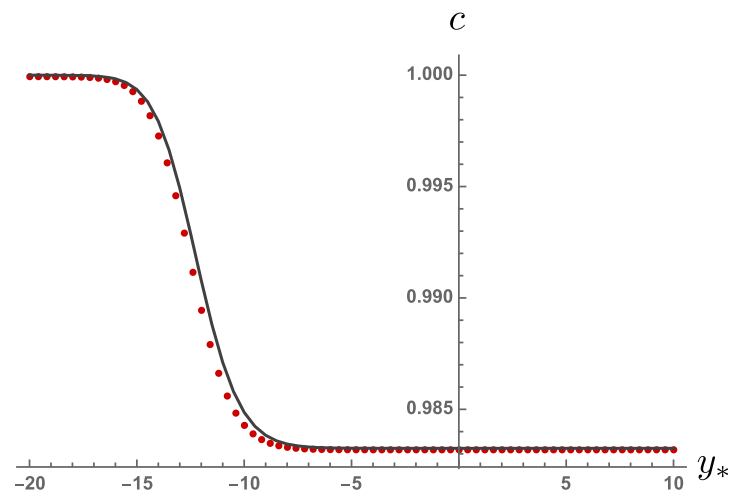

(a)

\section{DISCUSSION}

We investigated several different types of RG flow that can appear in a two-dimensional deformed CFT. To realize such RG flows holographically, we took into account a three-dimensional dual gravity theory with a specific scalar potential that allows many local extrema. Because of the invariance under $\phi \rightarrow-\phi$, the potential usually allows a local extremum at $\phi=0$ which was identified with the UV fixed point of the dual field theory. Near this UV fixed point, if we restrict for the scalar field to have a mass only in the range of $-1<m^{2} R_{\mathrm{uv}}^{2}<0$, the local extremum at $\phi=0$ becomes a local maximum or an unstable equilibrium point and, on the dual field theory side, the corresponding dual scalar operator becomes a relevant operator. Although the effect of a relevant operator is negligible in the UV regime, it causes a nontrivial RG flow and seriously modifies the IR physics. On the dual gravity side, the RG flow caused by a relevant scalar operator can be matched to the rolling of the bulk scalar field. Since the potential considered here allows a local minimum near the local maximum defined only except for $a=0$, the scalar field naturally rolls down to a local minimum that corresponds to

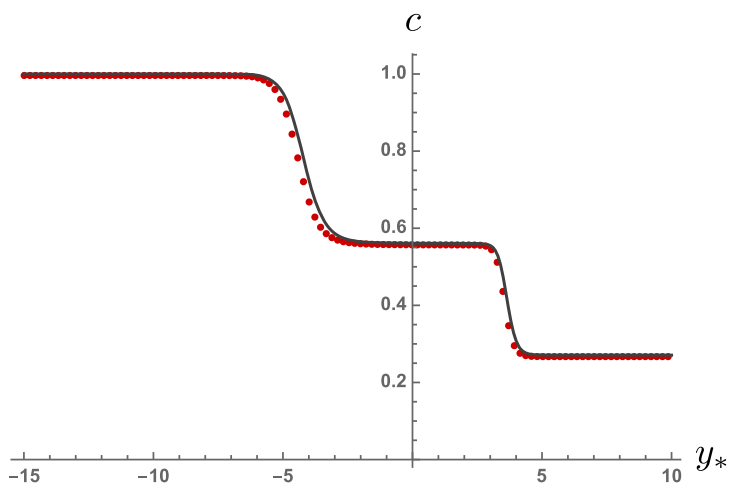

(b)

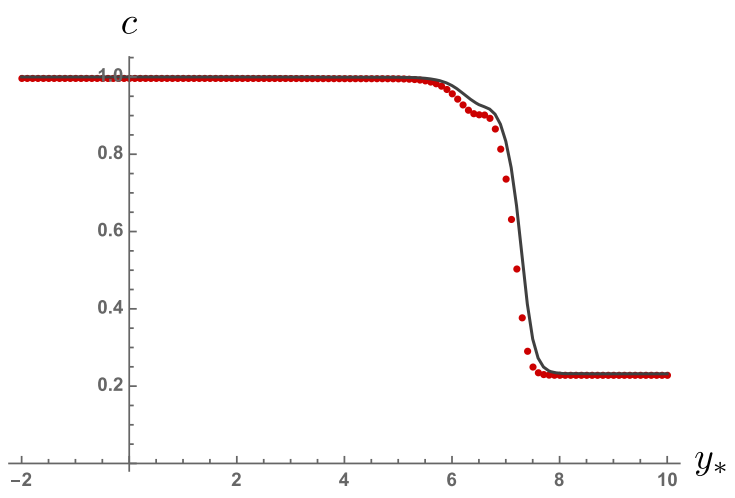

(c)

FIG. 9. Comparing holographic $c$ functions: (a) standard RG, (b) staircase RG, and (c) bouncing RG, where the red dotted line represents the result of (2.6) and the black line represents the entanglement $c$ function (4.6). Here we fix the central charge to be 1 at the UV fixed point. 
a new IR fixed point of the deformed field theory. As a consequence, the rolling of the scalar field from the unstable to a new stable equilibrium point describes the RG flow of the dual field theory from the UV to IR fixed points. From the field theory point of view, since this RG flow is highly nonperturbative, it is usually a very difficult task to understand the details of the RG flow in the entire region of the energy scale. However, there is still a chance to investigate the nonperturbative feature of the RG flow by using the holography. In the present work, we have studied the possible RG flows of the quantum field theory deformed by a relevant scalar operator. Interestingly, we showed that the toy model we considered leads to several different types of the RG flow relying on the value of the intrinsic parameter. The resulting RG flows can be summarized as follows:

(i) For $a>0$, the RG flow of the dual field theory is described by the standard RG flow, in which the $\beta$ function is always negative and does not oscillate at the intermediate energy scale. From this result, we can see that the coupling constant of the dual field theory increases monotonically along with the RG flow, while the $c$ function corresponding to the degrees of freedom monotonically decreases.

(ii) For $a=0$, we showed that there is no local minimum near the local maximum corresponding to the UV fixed point. This implies that the RG flow is not terminated due to the absence of the IR fixed point. Therefore, the RG flow for $a=0$ becomes an IR incomplete RG flow.

(iii) For $a<0$, a new IR fixed point appears again so that the corresponding RG flow is IR complete. In this parameter region, the resulting RG flow shows two different flowing behaviors from the previous standard RG flow. When the absolute value of $a$ is very small, the staircase RG flow appears. The $\beta$ function of the staircase RG flow is always negative except for two fixed points, which is similar to the previous standard RG flow. Unlike the standard RG flow, however, the $\beta$ function of the staircase RG flow oscillates. Therefore, the coupling constant of the staircase RG flow increases monotonically along with the RG flow but repeats the fast and slow increasing due to the oscillation of the $\beta$ function. For a large absolute value of $a$, the bouncing RG flow occurs. In general, the bouncing RG flow has two branching solutions. One has a positive $\beta$ function, while the other branch has a negative value. At the bouncing points, the RG flow changes the branch with changing the sign of the $\beta$ function. On the dual field theory side, this feature shows that the coupling constant increasing in one branch becomes decreasing in the other branch after passing through the bouncing point. As a result, the interaction strength of the bouncing RG flow does not increase monotonically along with the RG flow. We finally showed that the number of the bouncing points in the bouncing RG flow increases as the absolute value of $a$ increases.

We also studied the $c$ function relying on the energy scale by using the holographic entanglement entropy technique. In a variety of the RG flows we have found a nontrivial $\beta$ function that determines the strength of the coupling constant. For the bouncing RG flow, since the $\beta$ function has a positive value at an intermediate energy scale, the interaction strength can decrease in the course of the RG flow. At this energy scale, it would be interesting to ask how the $c$ function behaves. By applying the holographic entanglement entropy technique, we studied the change of the $c$ function for various RG flows we found. Intriguingly, we numerically showed that the $c$ function always decreases along with the RG flow regardless of the type of the RG flow. Even in the bouncing RG flow that allows the decreasing coupling constant, for example, the $c$ function monotonically decreases with satisfying the $c$ theorem. In the present work, we focused on only the two-dimensional deformed CFT. However, it would be more interesting to understand the possible RG flows that can appear in higher dimensional deformed CFTs by applying the methods used in this work. We hope to report more interesting results in future works.

\section{ACKNOWLEDGMENTS}

We are grateful to Yunseok Seo for insightful discussions. This work was supported by Basic Science Research Program through NRF Grant No. NRF-2016R1D1A1B03932371 and by Mid-career Researcher Program through the National Research Foundation of Korea Grant No. NRF2019R1A2C1006639. J. L. was supported by Basic Science Research Program through the National Research Foundation of Korea funded by the Ministry of Education Grant No. NRF-2018R1A6A3A11049655. 
[1] E. Witten, Adv. Theor. Math. Phys. 2, 253 (1998).

[2] J. M. Maldacena, Int. J. Theor. Phys. 38, 1113 (1999); Adv. Theor. Math. Phys. 2, 231 (1998).

[3] S. S. Gubser, I. R. Klebanov, and A. M. Polyakov, Nucl. Phys. B636, 99 (2002).

[4] J. M. Maldacena and J. G. Russo, J. High Energy Phys. 09 (1999) 025.

[5] A. B. Zamolodchikov, Pis'ma Zh. Eksp. Teor. Fiz. 43, 565 (1986) [JETP Lett. 43, 730 (1986)].

[6] P. Calabrese and J. L. Cardy, J. Stat. Mech. (2004) P06002.

[7] D. L. Jafferis, I. R. Klebanov, S. S. Pufu, and B. R. Safdi, J. High Energy Phys. 06 (2011) 102.

[8] J. L. Cardy, Phys. Lett. B 215, 749 (1988).

[9] Z. Komargodski and A. Schwimmer, J. High Energy Phys. 12 (2011) 099.

[10] Z. Komargodski, J. High Energy Phys. 07 (2012) 069.

[11] R. C. Myers and A. Sinha, J. High Energy Phys. 01 (2011) 125.

[12] I. R. Klebanov, S. S. Pufu, and B. R. Safdi, J. High Energy Phys. 10 (2011) 038.

[13] S. Ryu and T. Takayanagi, Phys. Rev. Lett. 96, 181602 (2006).

[14] S. Ryu and T. Takayanagi, J. High Energy Phys. 08 (2006) 045.

[15] S. Gubser, I. Klebanov, and A. Polyakov, Phys. Lett. B 428, 105 (1998).

[16] E. Witten, Adv. Theor. Math. Phys. 2, 505 (1998).

[17] H. Casini and M. Huerta, Phys. Lett. B 600, 142 (2004).

[18] H. Casini and M. Huerta, J. Phys. A 40, 7031 (2007).

[19] T. Albash and C. V. Johnson, J. High Energy Phys. 12 (2012) 095.

[20] I. R. Klebanov, T. Nishioka, S. S. Pufu, and B. R. Safdi, J. High Energy Phys. 07 (2012) 001.

[21] S. Cremonini and X. Dong, Phys. Rev. D 89, 065041 (2014).

[22] T. Faulkner, J. High Energy Phys. 05 (2015) 033.

[23] C. Park, Adv. High Energy Phys. 2014, 565219 (2014).

[24] C. Park, Phys. Rev. D 93, 086003 (2016).

[25] H. Casini, E. Teste, and G. Torroba, J. High Energy Phys. 03 (2016) 033.

[26] K.-S. Kim and C. Park, Phys. Rev. D 93, 121702 (2016).

[27] P. Bueno and W. Witczak-Krempa, Phys. Rev. D 95, 066007 (2017).
[28] K.-S. Kim, M. Park, J. Cho, and C. Park, Phys. Rev. D 96, 086015 (2017).

[29] K.-S. Kim and C. Park, Phys. Rev. D 95, 106007 (2017).

[30] K.-S. Kim, S. B. Chung, C. Park, and J.-H. Han, Phys. Rev. D 99, 105012 (2019).

[31] O.-K. Kwon, D. Jang, Y. Kim, and D. D. Tolla, J. High Energy Phys. 05 (2018) 009.

[32] J. K. Ghosh, E. Kiritsis, F. Nitti, and L. T. Witkowski, J. High Energy Phys. 05 (2018) 034.

[33] R. Narayanan, C. Park, and Y.-L. Zhang, Phys. Rev. D 99, 046019 (2019).

[34] C. Park, D. Ro, and J. Hun Lee, J. High Energy Phys. 11 (2018) 165.

[35] D. Dudal and S. Mahapatra, J. High Energy Phys. 07 (2018) 120.

[36] N. Deger, A. Kaya, E. Sezgin, and P. Sundell, Nucl. Phys. B573, 275 (2000).

[37] N. S. Deger, J. High Energy Phys. 11 (2002) 025.

[38] E. Kiritsis, F. Nitti, and L. Silva Pimenta, Fortschr. Phys. 65, 1600120 (2017).

[39] R. C. Myers and A. Sinha, Phys. Rev. D 82, 046006 (2010).

[40] D. Z. Freedman, S. S. Gubser, K. Pilch, and N. P. Warner, Adv. Theor. Math. Phys. 3, 363 (1999).

[41] J. de Boer, Fortschr. Phys. 49, 339 (2001).

[42] K. Skenderis and P. K. Townsend, Phys. Rev. D 74, 125008 (2006).

[43] I. Papadimitriou, J. High Energy Phys. 08 (2011) 119.

[44] I. R. Klebanov and E. Witten, Nucl. Phys. B556, 89 (1999).

[45] E. Witten, arXiv:hep-th/0112258.

[46] I. Klebanov and A. Polyakov, Phys. Lett. B 550, 213 (2002).

[47] U. Grsoy, E. Kiritsis, F. Nitti, and L. Silva Pimenta, J. High Energy Phys. 10 (2018) 173.

[48] S. Weinberg, Phys. Rev. D 86, 105015 (2012).

[49] I. R. Klebanov and M. J. Strassler, J. High Energy Phys. 08 (2000) 052.

[50] F. A. Smirnov and A. B. Zamolodchikov, Nucl. Phys. B915, 363 (2017).

[51] M. Caselle, D. Fioravanti, F. Gliozzi, and R. Tateo, J. High Energy Phys. 07 (2013) 071.

[52] L. McGough, M. Mezei, and H. Verlinde, J. High Energy Phys. 04 (2018) 010.

[53] C. Park, Int. J. Mod. Phys. A 33, 1850226 (2018).

[54] S. Gukov, J. High Energy Phys. 01 (2016) 020.

[55] R. C. Myers and A. Singh, J. High Energy Phys. 04 (2012) 122. 